\title{
Cholinergic interneurons drive maladaptive changes in thalamostriatal circuitry after dopamine depletion
}

Tanimura A, Du Y, Kondapalli J, Wokosin DL, and Surmeier DJ. Cholinergic Interneurons Amplify Thalamostriatal Excitation of Striatal Indirect Pathway Neurons in Parkinson's Disease Models. Neuron. 2019; DOI: http://doi.org/10.1016/j.neuron.2018.12.004

Direct and indirect pathway spiny projection neurons in the striatum bidirectionally modulate the output nuclei of the basal ganglia [1]. An imbalance of these two pathways is a key contributor to the motor symptoms of Parkinson's disease (PD) [2]. In the classical model of striatal function, dopamine promotes movement by activating stimulatory D1 dopamine receptors on "direct" striatonigral spiny projection neurons (dSPNs) and inhibitory D2 receptors on "indirect" striatopallidal SPNs (iSPNs). In this model, dopamine loss tips the scales toward the movementsuppressing indirect pathway, resulting in hypokinetic PD symptoms.

SPNs are synaptically connected to many cell types. Beyond the direct impacts of dopamine loss on SPN function, dopamine loss initiates a cascade of circuit adaptations that can indirectly disrupt SPN function. These maladaptations are thought to be a significant cause of motor dysfunction in PD, but are poorly understood. A new study by Tanimura and colleagues advances understanding of circuit adaptations in PD, reporting a novel and behaviorally relevant adaptation mediated by parafascicular thalamostriatal projection neurons (PFn) and striatal cholinergic interneurons (ChI) [3]. Using a combination of pharmacologic, chemogenetic, and optogenetic techniques, they demonstrate that in the 6-OHDA PD model, PFn exhibit heightened connectivity with iSPNs, thereby enhancing iSPN activity. Interestingly, this effect requires ChI activity, as chemogenetic suppression of these cells significantly reduced the enhanced PFniSPN connectivity. Additional studies demonstrate that in 6-OHDA mice, ChIs potentiate PFn terminals that synapse on iSPNs via increased acetylcholine release acting upon $\alpha 6$-containing nicotinic acetylcholine receptors. Two causes were identified for this increased ACh release. One is the well-known loss of D2 autoreceptor-mediated ChI inhibition. Second, Tanimura and colleagues identified a novel source of ChI hyperactivity: an increase in the synaptic strength of PFn terminals onto ChI. In vivo data support the importance of these findings, demonstrating that chemogenetic suppression of ChIs or PFn neurons, or knockdown of $\alpha 6$ mRNA, reverses motor learning deficits in 6-OHDA mice.

In addition to defining a novel maladaptive pathway that disrupts the dSPN/iSPN balance, this work points to $\alpha 6$-containing nAChRs and downstream signaling events as a potential therapeutic target for PD. The mechanism underlying the increased strength of PFn synapses onto ChIs remains unknown, and will be an important goal for future studies. Considered broadly, this work emphasizes the importance of considering the effects of dopamine depletion on the wider striatal network to identify novel molecular targets for PD therapeutics.

This is the author manuscript accepted for publication and has undergone full peer review but has not been through the copyediting, typesetting, pagination and proofreading process, which may lead to differences between this version and the Version of Record. Please cite this article as doi: $10.1002 / \mathrm{mds} .27685$

This article is protected by copyright. All rights reserved. 
1. Nelson, A.B. and A.C. Kreitzer, Reassessing models of basal ganglia function and dysfunction. Annu Rev Neurosci, 2014. 37: p. 117-35.

2. Albin, R.L., A.B. Young, and J.B. Penney, The functional anatomy of basal ganglia disorders. Trends Neurosci, 1989. 12(10): p. 366-75.

3. Tanimura, A., et al., Cholinergic Interneurons Amplify Thalamostriatal Excitation of Striatal Indirect Pathway Neurons in Parkinson's Disease Models. Neuron, 2019.

This article is protected by copyright. All rights reserved. 